\title{
Dutasteride and Metformin Reduce the Growth of LNCaP Cells and Alter the SREBP-1 Pathway
}

\author{
Rickvinder Besla ${ }^{1}$, Natalie Venier ${ }^{1}$, Alexandra Colquhoun ${ }^{1}$, Neil E. Fleshner ${ }^{2}$, Laurence H. Klotz ${ }^{1}$, \\ and Vasundara Venkateswaran ${ }^{1, *}$ \\ ${ }^{1}$ Division of Urology, Sunnybrook Health Sciences Centre, Toronto, ON, Canada \\ ${ }^{2}$ Division of Urology, Princess Margaret Hospital, University Health Network, Toronto, ON, Canada
}

\begin{abstract}
Prostate cancer ( $\mathrm{PCa}$ ) is the second most prevalent cancer in men after lung cancer. Prostate cancer development and progression is associated with the dysregulation of a number of molecular pathways; hence, therapeutic strategies targeting such pathways bring great promise. Recently we have shown that metformin, the anti-diabetic drug, can inhibit tumor progression when combined with dutasteride, a 5-alpha-reductase inhibitor (5ARI). Interestingly, both metformin and dutasteride have been reported to alter the Sterol Regulatory Element Binding Proteins (SREBP) Fatty Acid Synthase (FASN) pathway. The SREBP pathway is involved with lipid and energy homeostasis. In our present study, we investigated if dutasteride in combination with metformin can reduce the proliferation of $\mathrm{LNCaP} \mathrm{PCa}$ cells, and whether this is mediated through the SREBP-1/FASN pathway. Human PCa cells were treated with either dutasteride $(0-100 \mu \mathrm{M})$ or metformin $(0-50 \mathrm{mM})$ alone or in combination. The treated cells were then incubated for up to 24 hours, and proliferation assessed using the MTS 3-(4,5-dimethylthiazol-2-yl)-5-(3-carboxymethoxyphenyl)-2-(4-sulfophenyl)-2H-tetrazolium) assay. Western blot analysis was performed on cell lysates to assess alterations in key signaling molecules including cleaved SREBP-1, FASN, AR, PSA, pAMPK and apoptotic markers. Results revealed that there was a significant $(\mathrm{p}<0.05)$ decrease in cellular proliferation of $\mathrm{LNCaP}$ cells treated with a combination of metformin and dutasteride, this effect being greater than either treatment alone. Treating LNCaP cells with both metformin and dutasteride reduced the expression of FASN, cleaved SREBP-1 and pro-caspase-3 with expression of cleaved PARP; suggesting a possible interaction between FASN and apoptosis. All treatments resulted in reductions in AR, PSA and an upregulation of pAMPK, with the highest expression seen in combination treatment. We report for the first time that metformin and dutasteride in combination can reduce the proliferation of androgen-sensitive cell line through the activation of p-AMPK, and SREBP1/FASN pathway and highlight the importance of targeting the SREBP-1 pathway, for improving future therapeutic strategies for prostate cancer.
\end{abstract}

Keywords: Metformin, Dutasteride, Prostate Cancer, SREBP-1, Proliferation, Apoptosis.

\section{INTRODUCTION}

Energy metabolism is a critical event in cancer development and progression [1-3]. In the early stages of carcinogenesis, energy processes are upregulated to meet the cells' demand and as a result there is an increase in fatty acid biosynthesis by the protein fatty acid synthase (FASN) [4,5]. Increased FASN expression has been shown to be highly upregulated in early stage prostate cancer, and further increased in invasive PCa, suggesting that FASN plays a major role in the initial stages of prostate tumorigenesis [6]. FASN expression is relatively low in human cells due to exogenous fatty acid usage from diet [7]. However, in prostate cancer,

*Address correspondence to this author at the Department of Surgery, University of Toronto, Division of Urology, Sunnybrook Health Sciences Centre, S-118B, 2075 Bayview Avenue, Toronto, Ontario, M4N 3M5 Canada; Tel: 416-480-6100; Ext: 3127; Fax: 416-480-5737;

E-mail: vasundara.venkateswaran@sunnybrook.ca the greater production of androgens indirectly influences the production of FASN through stimulating the sterol regulatory element binding protein (SREBP) pathway $[8,9]$. As this pathway has been shown to be integral for PCa progression, therapeutic strategies targeting the SREBP pathway may show promise.

Individual agents including the oral biguanide, metformin, and the $5 \alpha$-reductase inhibitor, dutasteride, have both been shown to reduce FASN expression in vitro and in vivo PCa models $[10,11]$. Dutasteride was originally demonstrated to reduce benign prostatic hyperplasia, through preventing the conversion of testosterone to the more potent androgen DHT [12]. However, recent studies have shown that dutasteride induces apoptosis in prostate cancer cells through a FASN-mediated pathway [10,13-15]. This newly found potential of dutasteride requires further investigation for this to be considered as a potent therapeutic option for PCa. 
One of the ways metformin exerts its anti-diabetic effects is by preventing gluconeogenesis through the activation of the AMP activated protein kinase (AMPK) pathway [16-20]. Metformin has also been shown to reduce the growth of colon cancer tumour growth in mice fed a high-fat diet [11]. Algire et al., found that metformin caused a reduction in SREBP-1 expression through the activation of AMPK, thereby lowering expression levels of FASN in vivo [11]. These findings have not been replicated using human prostate cancer $\mathrm{LNCaP}$ cells. Also there is no in vitro or in vivo studies to date that have elucidated the mechanism of action of these compounds in combination. The present study aims to delineate the mechanism of action of metformin and dutasteride on $\mathrm{LNCaP}$ cells in vitro.

We demonstrate that a combination of dutasteride and metformin causes a significant reduction in LNCaP cell viability and alter the FASN pathway, leading to the downregulation of androgen signaling and PSA expression.

\section{MATERIALS AND METHODS}

\section{Cell Culture}

The androgen-sensitive human prostate cancer cell line (LNCaP) was obtained from the American Type Tissue Collection Rockville, MD, USA. Cells were grown in RPMI1640 supplemented with L-glutamine, $10 \%$ fetal bovine serum, streptomycin and penicillin and incubated in a $37^{\circ} \mathrm{C}$ sterile environment. Cells were typically grown to $70-80 \%$ confluence and passaged 1:5.

\section{MTS Cell Proliferation Assay}

Cell proliferation was assessed using the MTS assay (Promega, Madison, WI, USA) Cells were first plated in a 96-well plate at a density of 5000 cells/well and left to adhere in standard media. After 48 hours, media was removed and cells were treated with either metformin and/or dutasteride for 24 hours as detailed below. Dutasteride (SantaCruz Biotechnology, Santa Cruz, CA, USA) was diluted in DMSO to a stock solution $(100 \mathrm{mM})$ then diluted to a range of concentrations (1-100 $\mu \mathrm{M})$. Metformin (Santa Cruz Biotechnology, Santa Cruz, CA, USA) was dissolved immediately before use in media to concentrations ranging from 0.01-50 mM. Controls were treated with vehicle alone. After 24 hours, a $20 \mu \mathrm{l}$ MTS solution was added to each well and left to incubate for 2 hours (as per protocol). The mitochondrial reductase enzyme in viable cells converted the tetrazolium salts into a coloured formazan product that was directly measured $(490 \mathrm{~nm})$ using an absorbance plate reader [21]. Each experiment was performed in triplicates.

\section{Western Blot Analysis}

LNCaP cells were plated and treated with metformin (10 $\mathrm{mM})$ and/or dutasteride $(70 \mu \mathrm{M})$ for 24 hours and lysed with NP-40 lysis buffer containing protease inhibitors. Various cell lysates was loaded in $12 \%$ SDS-PAGE gel at $20 \mu \mathrm{g}$ concentrations and protein transferred on to a PVC membrane over night and blocked with 5\% milk for 2 hours. Primary antibodies were incubated overnight with tris buffer containing $5 \%$ milk while phosphorylated proteins were incubated with tris buffer having $5 \%$ bovine serum albumin. Secondary antibodies were incubated with buffer in 5\% milk for 1 hour. Protein detection was performed with electrochemiluminescence (Pierce). Densitometry was performed using ImageJ (National institute of Health, Bethesda, Maryland, USA) for all proteins using their respective $\beta$-actin, while phosphorylated proteins were compared against its nonphosphorylated total. Quantification of each protein expression was represented relative to respective controls. Primary antibodies for Akt, AMPK, FASN, pAMPK $\alpha-\mathrm{Thr}^{172}$, PARP, Pro-caspase-3 were purchased from Cell Signaling Technologies (Danvers, MA, USA) and used in 1:1000 dilutions. Primary antibodies for SREBP-1C, AR, and PSA were purchased from Santa Cruz Biotechnology (Santa Cruz, CA, USA) and were used in 1:200 dilutions. Secondary antibodies were purchased from Promega (Madison, WI, USA) and used in 1:5000 dilutions. Each experiment was performed in triplicates.

\section{Statistical Analysis}

The unpaired Student's ' $t$ '-test (two-tailed) was performed to establish statistical significance for cellular proliferation studies and densitometry in all treatment groups.

\section{RESULTS}

\section{Metformin and Dutasteride Cause a Significant Reduc- tion in the Proliferation of LNCaP Cells In Vitro}

The effect of dutasteride on the proliferation of human LNCaP cells was evaluated using a dose range from 0-100 $\mu \mathrm{M}$. Treatment of LNCaP cells in culture with dutasteride for 24 hours resulted in a dose-dependent reduction in cellular proliferation, with a significant reduction observed at a concentration of $80 \mu \mathrm{M}(\mathrm{P}<0.05)$ (Fig. 1A). Metformin used with a dose range of $0-50 \mathrm{mM}$ caused a significant reduction in the proliferation of $\mathrm{LNCaP}$ cells starting at $10 \mathrm{mM}$ $(\mathrm{P}<0.05)$ (Fig. 1B). Based on these results, combinatorial studies with varying concentrations of both compounds were performed (data not shown). A concentration of $10 \mathrm{mM}$ of metformin and $70 \mu \mathrm{M}$ of dutasteride was chosen based on each drugs ability to cause a significant reduction in cellular proliferation of LNCaP cells as a monotherapy $(\mathrm{P}<0.05)$ and when combined compared to either drug alone $(\mathrm{P}<0.05)$ (Fig. 1C).

\section{A Combination of Metformin and Dutasteride on LNCaP Cells Alter Intracellular Signaling Pathways}

\section{AMPK Phosphorylation}

AMPK phosphorylation at Thr172 was used to measure activation of AMPK by Western blot (Fig. 2A). We observed increased phosphorylated AMPK when treated with metformin alone, indicating that the dosage of metformin used was adequate to activate AMPK in LNCaP cells. Such an increase was also observed when treated with dutasteride alone. A slight increase in phosphorylated AMPK was observed when metformin was combined with dutasteride compared to metformin alone.

\section{SREBP-1 Pathway}



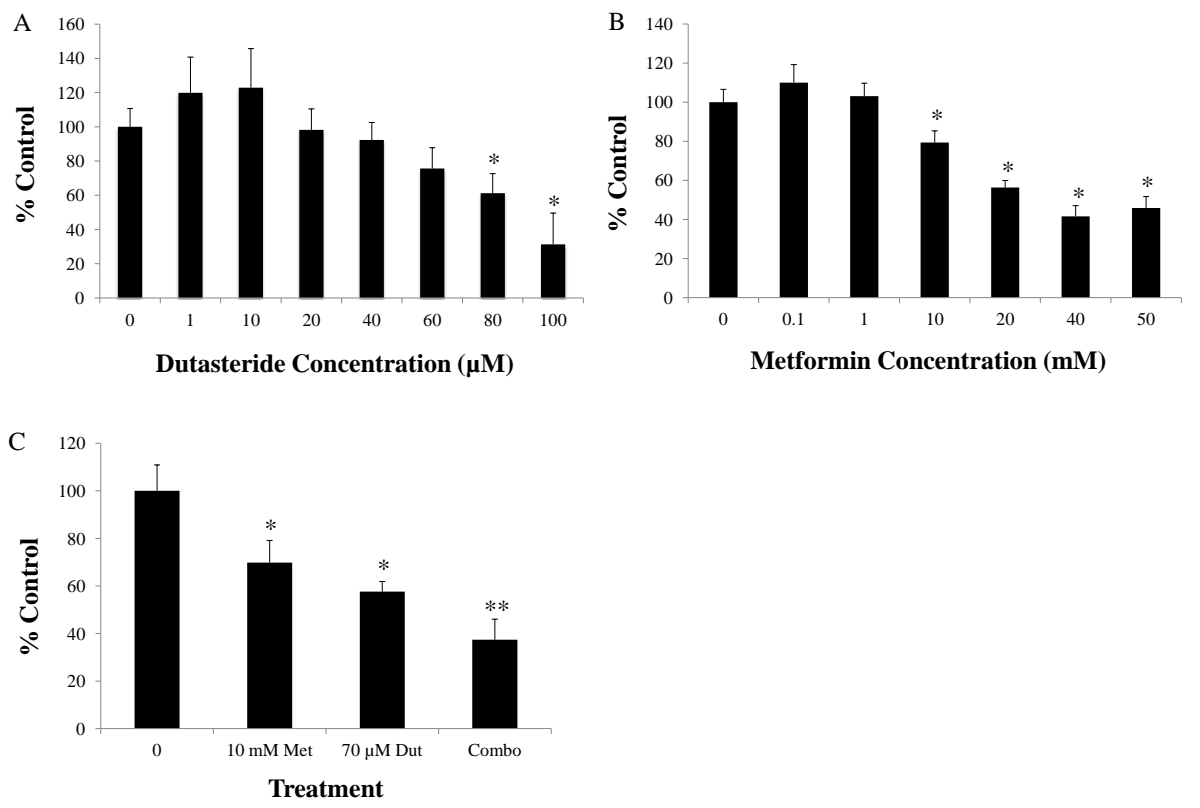

Fig. (1). MTS proliferation data for LNCaP cells after 24 hours. A) LNCaP cells were treated with a dose range of $0-100 \mu \mathrm{M}$ dutasteride. Significant reductions were seen after $80 \mu \mathrm{M}$. B) LNCaP cells were treated with a dose range of 0-50 mM metformin. Significant reductions were seen after $10 \mathrm{mM}$. C) LNCaP cells were treated with concentrations of $10 \mathrm{mM}$ metformin, $70 \mu \mathrm{M}$ dutasteride, and in combination. Combination therapy resulted in a significant reduction in proliferation compared to all groups. ${ }^{*} \mathrm{p}<0.05$ compared to control, ${ }^{* *} \mathrm{p}<0.05$ compared to all groups.

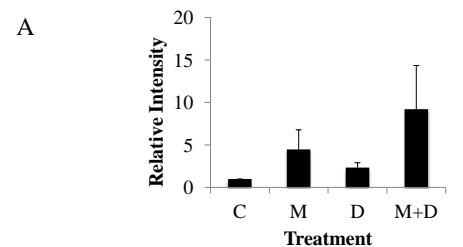

pAMPK-Thr ${ }^{172}$

AMPK Total

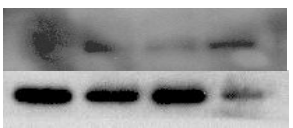

B

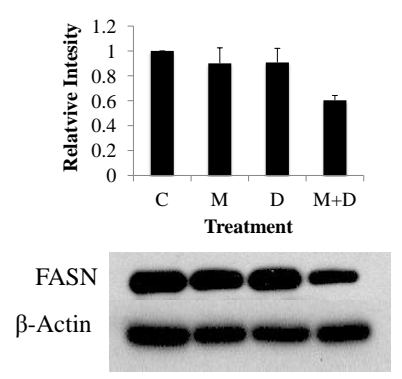

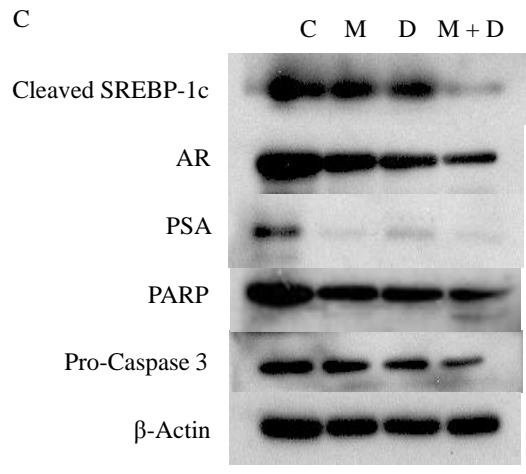

Fig. (2). Metformin and dutasteride in combination resulted in altered intracellular signaling pathways as determined by Western blot analysis. A) Metformin and dutasteride in combination increase the expression of phosphorylated AMPK compared to metformin alone in LNCaP cells. B) Metformin and dutasteride in combination resulted in the largest reductions in FASN compared to either treatment alone. C) Metformin and dutasteride in combination result in reductions of cleaved SREBP-1c, AR, PSA, Pro-caspase-3 and cleavage of PARP. (C - Control; M - Metformin; D - Dutasteride; M+D - Metformin + Dutasteride).

Expression of cleaved SREBP-1c and FASN was measured by Western blot analysis (Fig. 2 B,C). It was evident that the level of both cleaved SREBP-1c and FASN was significantly reduced with a combination of metformin and dutasteride treatment compared to either monotherapy.

\section{Androgen Receptor (AR) Changes}

Prostate specific antigen (PSA) is commonly used as a marker for prostate cancer progression and androgen receptor activity [21]. Western blot analysis was used to evaluate the alterations in protein expression caused by each treatment. All treatments resulted in a reduction in AR expression in $\mathrm{LNCaP}$ cells with the greatest effect seen with combined therapy (Fig. 2C). Subsequent reductions in PSA were also observed in all treatment groups (Fig. 2C). 


\section{Apoptotic Proteins}

Assessment of expression levels of apoptotic proteins through Western blot analysis was performed to delineate the cell death pathways activated after each treatment. Expression levels of cleaved PARP were only evident with combination therapy and not seen in monotherapy, this being directly correlated with reductions in the expression of procaspase-3 (Fig. 2C).

\section{DISCUSSION}

It is well established that androgens play a role in the growth and progression of prostate cancer [9]. In our study, we have found that dutasteride and metformin, in combination, reduce the proliferation of $\mathrm{LNCaP}$ cells greater than either drug alone. This anti-proliferative effect was found to correlate with a reduction in the expression of SREBP-1c and FASN signaling as well as the down-regulation of AR and PSA expression, demonstrating for the first time, a novel mechanism of action for these two agents in combination.

Early stages of PCa are heavily dependent on the interaction between endogenous androgens and the androgen receptor to drive growth and development [22]. When the androgen receptor translocates to the nucleus in $\mathrm{PCa}$ cells, the SREBP catalytic activating protein (SCAP) genes acts as an androgen response element (ARE) for the AR, leading to increased expression and enhancement of the translocation of SREBP1c resulting in the upregulation of FASN [8]. McInnes et al. [23] have shown that DHT can induce the expression of lipogenic gene, FASN, and decrease the basal and pharmacological activation of AMPK in 3T3-L1 adipocyte cells [23].
In our current study, we have found that dutasteride downregulates the expression of $\mathrm{AR}$, and upregulates the expression of pAMPK in LNCaP cells treated with dutasteride alone. Interestingly, this activation is further upregulated when combined with metformin, an established AMPK activator. One of the ways metformin has been shown to exert its anti-diabetic effects is by preventing gluconeogenesis through the activation of the AMP activated protein kinase (AMPK) pathway [16-19]. Based on these findings, we suspect that dutasteride inhibits the production of DHT, relieving the activation of AMPK by DHT, resulting in the up-regulation of activated AMPK (Fig. 3). Furthermore, we believe that the combined effect of both the DHT inhibition and the activation of AMPK caused a reduction in the expression of FASN in the group treated with a combination of dutasteride and metformin. This effect may be facilitated by the reduced expression in SREBP-1, which has recently been shown to regulate expression of AR [24]. This recent study by Huang et al showed that by genetically overexpressing or knocking down SREBP-1 in LNCaP cells resulted in a corresponding increase or decrease in AR expression [24]. Our data confirms the above reported findings where the greatest reduction in AR and PSA expression was seen with a combination group of dutasteride and metformin, with a corresponding reduction of SREBP-1. Further, the downregulation of FASN correlated with the induction of apoptosis as demonstrated through cleavage of PARP and a reduction pro-caspase-3 expression. This correlation between FASN inhibition and an apoptosis feedback effect has been previously reported [25-27]. This cell death pathway was not seen in cells treated with either monotherapy indicating that cell cycle arrest occurred, due to increases observed with inhibitory cell cycle marker p21 and reductions in cyclin D1

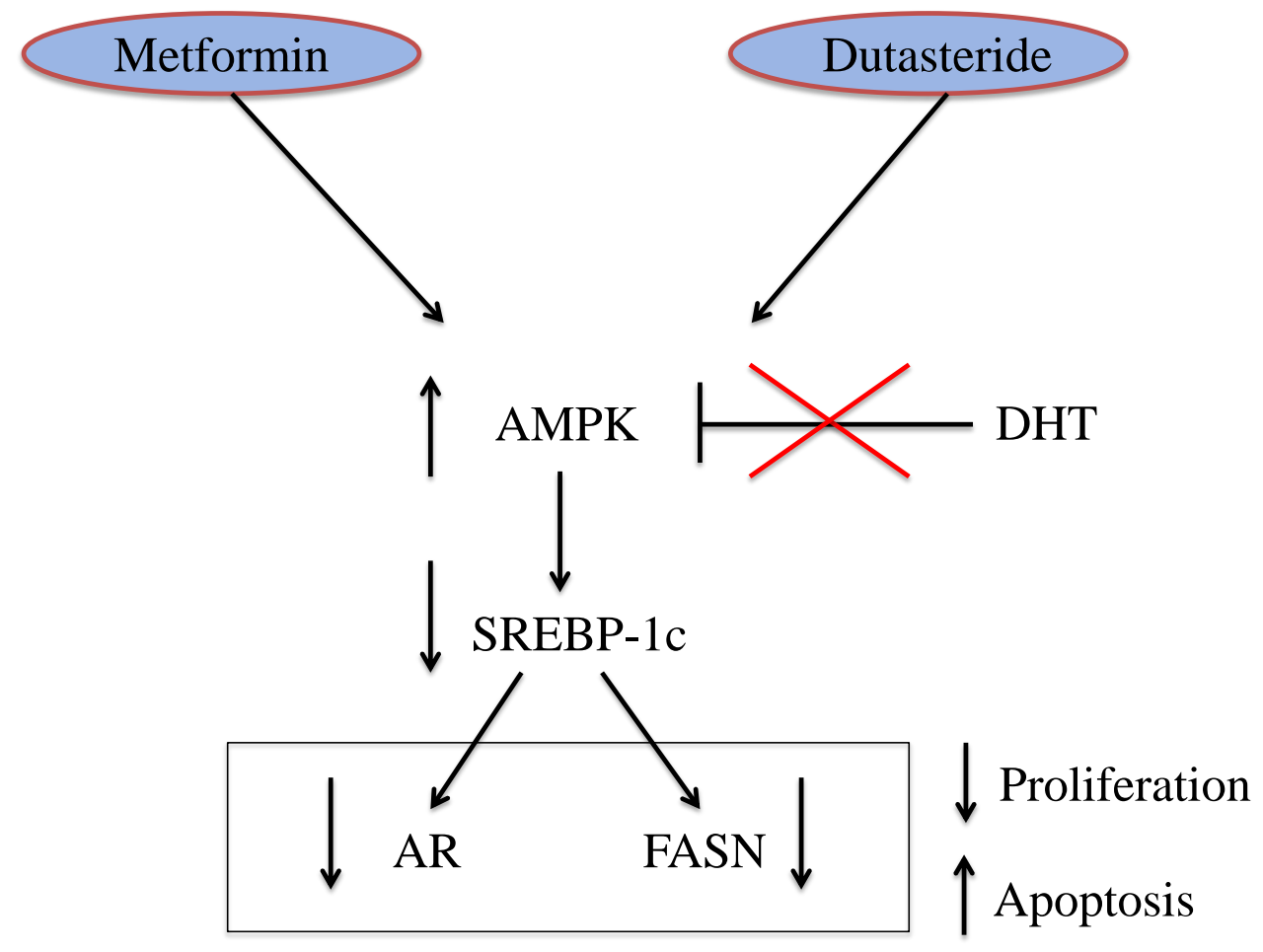

Fig. (3). The proposed mechanism by which metformin and dutasteride reduce proliferation and increase apoptosis in LNCaP cells. 
(Supplementary figure). Future studies using a FASN enzyme activity assays may be conducted for a better understanding of this pathway. Finally, alterations in SREBP-1c were seen in both treatments groups alone but did not result in reductions in FASN expression perhaps due to the specificity of the SREBP-1c antibody being used. Heemers et al. [8] states that immunohisto chemical techniques for detecting SREBP cannot differentiate between mature/cleaved and precursor SREBP proteins, suggesting that these results may not adequately represent the true activation of SREBP-1. However, future analysis of lysates for nuclear proteins may prove useful as SREBP-1c is involved with FASN transcription in the nucleus.

There are several caveats to this study. Firstly, LNCaP cells were cultured in media containing fetal bovine serum, which contains androgens; thus, a high dose of dutasteride was used to see the observed effect. These doses have not been previously reported, and may have had a cytotoxic effect on the LNCaP cells. Recently Mostaghel et al. [28] have shown that high dose dutasteride treatments were associated with fewer cancer incidences in patients with specific gene expressions. Although not directly correlating to our study, it showed an increased interest in the anti-neoplastic potential of dutasteride at high dosages $[14,15]$. Future studies are warranted examining the mechanism of action of these agents at lower concentrations.

In summary, we have shown reduced SREBP-1c, AR, and FASN expression in LNCaP cells when treated with a combination of high dose dutasteride and metformin, resulting in a reductions in cellular proliferation and an induction of apoptosis (Fig. 3). Interest in these compounds has been increased over the years. Several reports have shown greater type 1 and 2, 5a reductase expression in higher grade $\mathrm{PCa}$ [29] and due to the incidence of metabolic syndrome in $\mathrm{PCa}$ patients receiving ADT [30-32]. As specific alterations in intracellular pathways were observed with combination treatment compared to each compound alone, further investigation in this process is required with both androgen dependent and independent prostate cancer cells.

\section{CONFLICT OF INTEREST}

The authors confirm that this article content has no conflicts of interest.

\section{ACKNOWLEDGEMENTS}

Assistance from Rashpaul K Bhogal, Sunnybrook Health Sciences Centre for the Western blot analysis is kindly acknowledged.

\section{REFERENCES}

[1] Shaw R. Glucose metabolism and cancer. Curr Opin Cell Biol 2006; 18: 598-608.

[2] Bui T, Thompson CB. Cancer's sweet tooth. Cancer Cell 2006; 9: 419-20.

[3] Garber K. Energy deregulation: licensing tumors to grow. Science 2006; 312: 1158-9.

[4] Kuhajda FP. Fatty acid synthase and human cancer: new perspective on its role in tumor biology. Nutrition 2000; 16: 202-8.

[5] Menendez J, Lupu R. Fatty acid synthase and the lipogenic phenotype in cancer pathogenesis. Nat Rev Cancer 2007; 7: 763-77.
[6] Swinnen JV, Roskams T, Joniau S, et al. Overexpression of fatty acid synthase is an early and common event in the development of prostate cancer. Int J Cancer 2002; 98: 19-22.

[7] Weiss L, Hoffman GE, Schreiber R, et al. Fatty acid biosynthesis in man, a pathway of minor importance: purification, optimal assay conditions, and organ distribution of fatty-acid synthase. Biol Chem Hoppe Seyler 1986; 367: 905-12.

[8] Heemers HV, Verhoeven G, Swinnen JV. Androgen activation of the sterol regulatory element- binding protein pathway: current insights. Mol Endocrinol 2006; 20: 2265-77.

[9] Heemers HV, Maes B, Foufelle F, et al. Androgens stimulate lipogenic gene expression in prostate cancer cells by activation of the sterol regulatory element-binding protein cleavage activating protein/sterol regulatory element binding protein pathway. Mol Endocrinol 2001; 15: 1817-28.

[10] Schmidt LJ, Ballman KV, Tindall DJ. Inhibition of fatty acidy synthase activity in prostate cancer cells by dutasteride. Prostate 2007; 67: 1111-20.

[11] Algire C, Amrein L, Zakikhani M, et al. Metformin blocks the stimulative effect of a high-energy diet on colon carcinoma growth in vivo and is associated with reduced expression of fatty acid synthase. Endocr Relat Cancer 2010; 17: 351-60.

[12] Rittmaster RS. 5alpha-reductase Inhibitors. J Androl 1997; 18: 5827.

[13] Lazier CB, Thomas LN, Douglas RC, et al. Dutasteride, the dual $5 \alpha$-Reductase inhibitor, inhibits androgen action and promotes cell death in the $\mathrm{LNCaP}$ prostate cancer cell line. Prostate 2004; 58: 130-44.

[14] Biancolella M, Valentini A, Minella D, et al. Effects of dutasteride on the expression of genes related to androgen metabolism and related pathway in human prostate cancer cell lines. Invest New Drugs 2007; 25: 491-7.

[15] McCrohan A, Morrissey C, O'Keane C, et al. Effects of the dual $5 \alpha$ - reductase inhibitor dutasteride on apoptosis in primary cultures of prostate cancer epithelial cells and cell lines. Cancer 2006; 106: 2743-52.

[16] Stumvoll M, Nurjhan N, Perriello G, et al. Metabolic effects of metformin in non-insulin-dependent diabetes mellitus. N Engl J Med 1995; 333: 550-4.

[17] Hundal RS, Krssak M, Dufour S, et al. Mechanism by which metformin reduces glucose production in type 2 diabetes. Diabetes 2000; 49: 2063-9.

[18] Zhou G, Myers R, Li Y, et al. Role of amp-activated protein kinase in mechanism of metformin action. J Clin Invest 2001; 108: 116774.

[19] Sahra IB, Marchand-Brustel YL, Tanti JF, et al. Metformin in cancer therapy: a new perspective for an old antidiabetic drug? Mol Cancer Ther 2010; 9: 1092-9.

[20] Zadra G, Priolo C, Patnaik A, et al. New strategies in prostate cancer: targeting lipogenic pathways and the energy sensor AMPK. Clin Cancer Res 2010; 16: 3322-8.

[21] Venkateswaran V, Klotz LH, Fleshner NE. Selenium modulation of cell proliferation and cell cycle biomarkers in human prostate carcinoma cell lines. Cancer Res 2002; 62: 2540-5.

[22] Andriole GL, Bostwick DG, Brawley OW, et al. Effect of dutasteride on the risk of prostate cancer. N Engl J Med 2010; 362: 1192-202.

[23] McInnes KJ, Corbould A, Simpson ER, et al. Regulation of adenosine 5 monophosphate-activated protein kinase and lipogenesis by androgens contributes to visceral obesity in an estrogen-deficient state. Endocrinology 2006; 147: 5907-13.

[24] Huang W-C, Li X, Liu J, et al. Activation of androgen receptor, lipogenesis, and oxidative stress converged by SREBP-1 is responsible for regulating growth and progression of prostate cancer cells. Mol Cancer Res 2011; 10: 133-42.

[25] Schrijver E, Brusselmans K, Heyns W, et al. RNA interferencemediated silencing of the fatty acid synthase gene attenuates growth and induced morphological changes and apoptosis of LNCaP prostate cancer cells. Cancer Res 2003; 63: 3799-804.

[26] Zhou WP, Simpson J, McFadden JM, et al. Fatty acid synthase inhibition triggers apoptosis during $\mathrm{S}$ phase in human cancer cells. Cancer Res 2003; 63: 7330-7.

[27] Kuhajda F. Fatty acid synthase and cancer: new application of an old pathway. Cancer Res 2006; 66: 5977-80.

[28] Mostaghel EA, Geng L, Holcomb I, et al. Variability in the androgen response of prostate epithelium to $5 \alpha$-reductase inhibition: im- 
plication for prostate cancer chemoprevention. Cancer Res 2010; 70: 1286-95.

[29] Thomas LN, Douglas RC, Lazier CB, et al. Levels of $5 \alpha$-reductase Type 1 and Type 2 are increased in localized high grade compared to low grade prostate cancer. J Urol 2008; 179: 147-51.

[30] Saylor PF, Smith MR. Metabolic complication of androgen deprivation therapy for prostate cancer. J Urol 2009; 181: 1998-2008.
[31] Flanagan J, Gray PK, Hahn N, et al. Presence of the metabolic syndrome is associated with shorter time to castration-resistant prostate cancer. Ann Oncol 2010; 22: 801-7.

[32] Clements A, Gao B, Yeap SHO, et al. Metformin in prostate cancer: two for the price of one. Ann Oncol 2011; 22(12): 1-5.

Received: December 17, 2012

Revised: December 17, 2012

Accepted: December 19, 2012

(C) Besla et al.; Licensee Bentham Open.

This is an open access article licensed under the terms of the Creative Commons Attribution Non-Commercial License (http://creativecommons.org/licenses/ by-nc/3.0/) which permits unrestricted, non-commercial use, distribution and reproduction in any medium, provided the work is properly cited. 